\title{
INVERSE LIMITS WITH COUNTABLY MARKOV INTERVAL FUNCTIONS
}

\author{
MatevŽ Črepnjak AND TJaša Lunder
}

University of Maribor, Slovenia

\begin{abstract}
We introduce countably Markov interval functions and show that two inverse limits with countably Markov interval bonding functions are homeomorphic if the functions follow the same pattern. This result presents a generalization of results of S. Holte, and I. Banič and T. Lunder.
\end{abstract}

\section{INTRODUCTION}

S. Holte proved that two inverse limits with Markov interval bonding functions (with respect to a finite set $A$, usually referred to as a Markov partition) are homeomorphic, if the Markov interval bonding functions are surjective and follow the same pattern, see [3]. A generalization of Markov interval maps was introduced in [1], where the authors defined so-called generalized Markov interval functions (with respect to a finite set $A$ which generalizes the notion of Markov partition). They are a non-trivial generalization of single-valued mappings from an interval $I$ to $I$ to set-valued functions from $I$ to $2^{I}$. In [1], the authors proved that also in this more general case, two inverse limits with generalized Markov interval bonding functions are homeomorphic, if the bonding functions are surjective and follow the same pattern.

In this paper we extend the notion of generalized Markov interval functions (as well as Markov interval functions) by introducing so-called countably Markov interval functions with respect to an infinite countable set $A$. As the main result of the paper we show that two inverse limits with countably

2010 Mathematics Subject Classification. 54F50, 37B45, 54C60.

Key words and phrases. Inverse limits, upper semicontinuous functions, countably Markov interval functions. 
Markov interval bonding functions are homeomorphic, if the bonding functions follow the same pattern. This improves the above mentioned results since the surjectivity of bonding functions is not required.

We point out that there already exist many examples of functions that have already been studied intensively in various areas for different reasons, and can now be interpreted as such countably Markov interval functions:

1. (skew) tent functions can be interpreted as countably Markov interval functions;

2. Bennet's function, see [6, p. 17] (a more detailed description of this function is given in Example 3.10);

3. a subfamily of Kelly's irreducible functions, see [7].

\section{Definitions And NOtATion}

Our definitions and notation mostly follow $[1,4,10]$.

A set is countable if it is finite or of the cardinality $\aleph_{0}$.

For a given set $A$ the number of elements of $A$ is denoted by $|A|$.

The Cantor - Bendixson derivative of a subset $A$ of a topological space $X$ is the set of all limit points of the set $A$. We denote the derivate set of the set $A$ by $A^{\prime}$.

A map is a continuous function. In the case where $f: \mathbb{R} \rightarrow \mathbb{R}$ is a map and $a \in \mathbb{R}$, we use $\lim _{x \downarrow a} f(x)$ to denote the right-hand limit and $\lim _{x \uparrow a} f(x)$ to denote the left-hand limit of the function $f$ at the point $a$ (for more details see $[11$, p. 83-95]). In Section 3 we define a generalization of this notion to limits of set-valued functions.

We use $\pi_{1}, \pi_{2}: X \times X \rightarrow X$ to denote the standard projections $\pi_{1}(x, y)=$ $x$ and $\pi_{2}(x, y)=y$.

For a metric space $(X, d)$, for $r>0$ and for $a \in X, B_{r}(a)=\{x \in$ $X \mid d(x, a)<r\}$ denotes an open ball in $X$.

For a compact metric space $X$, we denote by $2^{X}$ the set of all nonempty closed subsets of $X$.

If $f: X \rightarrow 2^{Y}$ is a function, then the graph of $f, \Gamma(f)$, is defined as $\Gamma(f)=\{(x, y) \in X \times Y \mid y \in f(x)\}$.

A function $f: X \rightarrow 2^{Y}$ has a surjective graph, if for each $y \in Y$ there is an $x \in X$, such that $y \in f(x)$.

Let $f: X \rightarrow 2^{Y}$ be a function. If for each open set $V \subseteq Y$, the set $\{x \in X \mid f(x) \subseteq V\}$ is open in $X$, then $f$ is an upper semicontinuous function (abbreviated u.s.c.) from $X$ to $2^{Y}$.

The following theorem is a well-known characterization of u.s.c. functions between compact metrics spaces (for example, see [4, p. 120, Theorem 2.1]).

TheOREM 2.1. Let $X$ and $Y$ be compact metric spaces and $f: X \rightarrow 2^{Y}$ a function. Then $f$ is u.s.c. if and only if its graph $\Gamma(f)$ is closed in $X \times Y$. 
If $F: X \rightarrow 2^{Y}$ is a u.s.c. function, where for each $x \in X$, the image $F(x)$ is a singleton in $Y$; then we can interpret it as a single-valued continuous function. Obviously, for any continuous function $f: X \rightarrow Y$, the function $F: X \rightarrow 2^{Y}$, defined by $F(x)=\{f(x)\}$, is an u.s.c. function. In this special case, we say that $F$ is injective if $f$ is injective.

Let $f: X \rightarrow 2^{Y}$ be a function. Then we say that $f$ is single-valued at some point $x \in X$ if $f(x)$ consists of a single point. We also say that $f$ is single-valued on some subspace $Z \subseteq X$ if the above holds for each $x \in Z$.

Let $A$ be a subset of $X$ and let $f: X \rightarrow 2^{Y}$ be a function. The restriction of $f$ on the set $A,\left.f\right|_{A}$, is the function from $A$ to $2^{Y}$ such that $\left.f\right|_{A}(x)=f(x)$ for every $x \in A$.

A sequence $\left\{X_{k}, f_{k}\right\}_{k=1}^{\infty}$ of compact metric spaces $X_{k}$ and u.s.c. functions $f_{k}: X_{k+1} \rightarrow 2^{X_{k}}$, is an inverse sequence with u.s.c. bonding functions.

The inverse limit of an inverse sequence $\left\{X_{k}, f_{k}\right\}_{k=1}^{\infty}$ with u.s.c. bonding functions is defined as the subspace of $\prod_{k=1}^{\infty} X_{k}$ of all points $\left(x_{1}, x_{2}, \ldots\right)$, such that $x_{k} \in f_{k}\left(x_{k+1}\right)$ for each $k$. The inverse limit of an inverse sequence $\left\{X_{k}, f_{k}\right\}_{k=1}^{\infty}$ is denoted by $\lim _{\longleftarrow}\left\{X_{k}, f_{k}\right\}_{k=1}^{\infty}$.

In this paper we deal only with the case when for each $k, X_{k}$ is a closed interval $I=[x, y]$ and $f_{k}: I \rightarrow 2^{I}$. So, we denote the inverse limit simply by $\lim _{\longleftarrow}\left\{I, f_{k}\right\}_{k=1}^{\infty}$.

The concept of inverse limits of inverse sequences with u.s.c. bonding functions (also known as generalized inverse limits) was introduced by Mahavier in [8] and later by Ingram and Mahavier in [4]. Since then, inverse limits have appeared in many papers (more references can be found in $[5,6]$ ).

\section{Countably Markov interval functions}

In this section we introduce countably Markov interval functions and show some of their properties.

There are many results about limits of sequences of sets in metric spaces, for examples see $[10$, p. 56] or $[9$, p. 17], where other references can be found. In the beginning of this section, we use a generalization of the above results about limits of sequences of sets, by dealing with limits of set-valued functions $f$, i.e. the left-hand $\operatorname{limit}_{\operatorname{Lim}_{t \uparrow a}} f(t)$ and the right-hand $\operatorname{limit}_{\operatorname{Lim}} \operatorname{Lim}_{t \downarrow} f(t)$. They are defined in such a way that in the cases where $f$ can be interpreted as a single-valued function, the above limits behave as standard limits for singlevalued functions (if they exist).

Definition 3.1. Let $f: I=[x, y] \rightarrow 2^{I}$ be a set-valued function. We define the left-hand limit and the right-hand limit of $f$ at a point $a \in I$ as follows:

$$
\begin{aligned}
\operatorname{Lim}_{t \uparrow a} f(t)= & \{s \in I \mid \text { for each } \varepsilon>0 \text { there exists } a z \in(a-\varepsilon, a) \\
& \text { such that } \left.(\{z\} \times f(z)) \cap B_{\varepsilon}(a, s) \neq \emptyset\right\} .
\end{aligned}
$$


$\operatorname{Lim}_{t \downarrow a} f(t)=\{s \in I \mid$ for each $\varepsilon>0$ there exists a $z \in(a, a+\varepsilon)$ such that $\left.(\{z\} \times f(z)) \cap B_{\varepsilon}(a, s) \neq \emptyset\right\}$.

REMARK 3.2. If $f: I=[x, y] \rightarrow 2^{I}$ is a set-valued function, then the limits $\operatorname{Lim}_{t \uparrow x} f(t)$ and $\operatorname{Lim}_{t \downarrow y} f(t)$ are empty sets.

EXAMPLE 3.3. Let the set-valued function $f:[0,1] \rightarrow 2^{[0,1]}$ be defined by its graph, which is the union of the following sets:

1. the straight line segment with endpoints $\left(\frac{1}{2}-\frac{1}{2 n}, \frac{1}{3}\right)$ and $\left(\frac{1}{2}-\frac{1}{2 n+1}, \frac{2}{3}\right)$ for each positive integer $n$,

2. the straight line segment with endpoints $\left(\frac{1}{2}, 0\right)$ to $\left(\frac{1}{2}, 1\right)$,

3. the straight line segment with endpoints $\left(\frac{1}{2}, \frac{4}{5}\right)$ to $(1,1)$, see Figure 1.

The left-hand limit of $f$ at the point $\frac{1}{2}$ is $\operatorname{Lim}_{t \uparrow \frac{1}{2}} f(t)=\left[\frac{1}{3}, \frac{2}{3}\right]$ and the right-hand limit is $\operatorname{Lim}_{t \downarrow \frac{1}{2}} f(t)=\left\{\frac{4}{5}\right\}$.

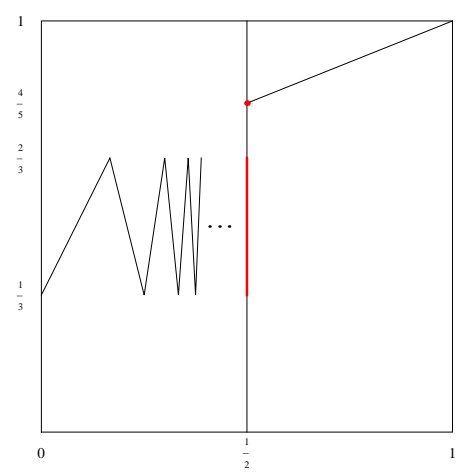

Figure 1. The graph of $f$ from Example 3.3.

ExAmple 3.4. Let $g: I=[0,1] \rightarrow 2^{I}$ be defined by its graph, $\Gamma(g)=$ $\left(\left[0, \frac{1}{2}\right) \times[0,1]\right) \cup\left(\left[\frac{1}{2}, 1\right] \times\left\{\frac{1}{2}\right\}\right)$, as seen in Figure 2. The left-hand limit of $g$ at the point $\frac{1}{2}$ is $\operatorname{Lim}_{t \uparrow \frac{1}{2}} g(t)=[0,1]$. The right-hand limit of $g$ at the point $\frac{1}{2}$ is $\operatorname{Lim}_{t \downarrow \frac{1}{2}} g(t)=\left\{\frac{1}{2}\right\}$.

The following auxiliary results mostly follow directly from Definition 3.1 and are easy to prove. For the completeness of the paper, we give the proofs anyway.

Lemma 3.5. Let $x, y \in \mathbb{R}, x<y$. Let $f: I=[x, y] \rightarrow 2^{I}$ be a u.s.c. function and $a \in I$. The following two statements hold true.

1. $\operatorname{Lim}_{t \uparrow a} f(t) \subseteq f(a)$ and $\operatorname{Lim}_{t \uparrow a} f(t)$ is closed in $f(a)$; if $a \neq x$ then $\operatorname{Lim}_{t \uparrow a} f(t) \neq \emptyset$. 


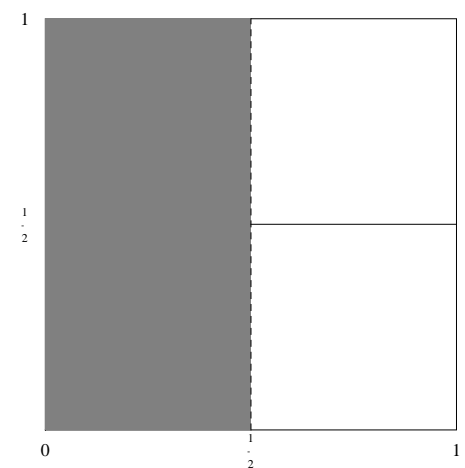

FiguRE 2. The graph of $g$ from Example 3.4.

2. $\operatorname{Lim}_{t \downarrow a} f(t) \subseteq f(a)$ and $\operatorname{Lim}_{t \downarrow a} f(t)$ is closed in $f(a)$; if $a \neq y$ then $\operatorname{Lim}_{t \downarrow a} f(t) \neq \emptyset$.

Proof. 1. If $a=x$ then $\operatorname{Lim}_{t \uparrow a} f(t)=\emptyset$ and therefore the claim holds true.

If $a \neq x$, then $\operatorname{Lim}_{t \uparrow a} f(t) \neq \emptyset-$ this is easily seen since $[x, y]$ is a compact metric space. Now suppose that $\operatorname{Lim}_{t \uparrow a} f(t) \nsubseteq \subseteq f(a)$. This means that there exists a point $(a, s) \in\{a\} \times\left(\operatorname{Lim}_{t \uparrow a} f(t) \backslash f(a)\right)$. From the definition of $\operatorname{Lim}_{t \uparrow a} f(t)$ it follows that there exists a convergent sequence $\left\{\left(x_{i}, s_{i}\right)\right\}_{i=1}^{\infty} \in \Gamma(f) \subseteq[x, y] \times[x, y]$ with the limit $(a, s)$. Since $s_{i} \in f\left(x_{i}\right)$ for each $i$ and $s \notin f(a)$, it follows that the graph of $f$ is not closed in $[x, y] \times[x, y]$ - a contradiction.

Now we prove that $\operatorname{Lim}_{t \uparrow a} f(t)$ is closed in $f(a)$.

Let $\left\{\left(a, x_{i}\right)\right\}_{i=1}^{\infty}$ be a convergent sequence of elements in $\operatorname{Lim}_{t \uparrow a} f(t)$, with the limit $\left(a, x^{\prime}\right) \in\{a\} \times f(a)$. We prove that $\left(a, x^{\prime}\right) \in\{a\} \times \operatorname{Lim}_{t \uparrow a} f(t)$.

Let $\varepsilon>0$. Since $\left(a, x^{\prime}\right)$ is the limit of $\left\{\left(a, x_{i}\right)\right\}_{i=1}^{\infty}$, there exists an $i_{0}$ such that $\left(a, x_{i_{0}}\right) \in B_{\frac{\varepsilon}{2}}\left(\left(a, x^{\prime}\right)\right) \subset[x, y] \times[x, y]$. Since $\left(a, x_{i_{0}}\right) \in\{a\} \times \operatorname{Lim}_{t \uparrow a} f(t)$, there exists a $z \in\left(a-\frac{\varepsilon}{2}, a\right)$ such that $(\{z\} \times f(z)) \cap B_{\frac{\varepsilon}{2}}\left(\left(a, x_{i_{0}}\right)\right) \neq \emptyset$. Therefore we can choose a point $\left(z, z^{\prime}\right)$ from $(\{z\} \times f(z)) \cap B_{\frac{\varepsilon}{2}}\left(\left(a, x_{i_{0}}\right)\right)$. Then

$$
d\left(\left(z, z^{\prime}\right),\left(a, x^{\prime}\right)\right) \leq d\left(\left(z, z^{\prime}\right),\left(a, x_{i_{0}}\right)\right)+d\left(\left(a, x_{i_{0}}\right),\left(a, x^{\prime}\right)\right) \leq \frac{\varepsilon}{2}+\frac{\varepsilon}{2}=\varepsilon .
$$

We have found a $z \in\left(a-\frac{\varepsilon}{2}, a\right) \subseteq(a-\varepsilon, a)$, such that $(\{z\} \times f(z)) \cap B_{\varepsilon}\left(\left(a, x^{\prime}\right)\right) \neq$ $\emptyset$.

2. A similar arguing gives that $\operatorname{Lim}_{t \downarrow a} f(t)$ is a closed subset of $f(a)$ and if $a \neq y$ then $\operatorname{Lim}_{t \downarrow a} f(t) \neq \emptyset$.

Lemma 3.6. Let $x, y \in \mathbb{R}, x<y$. Let $f: I=[x, y] \rightarrow 2^{I}$ be a u.s.c. function. If $f(a)$ is connected for each $a \in I$, then the following statements hold true for each $a \in I$. 
1. If $a \neq x$, then $\operatorname{Lim}_{t \uparrow a} f(t)$ is nonempty and connected.

2. If $a \neq y$, then $\operatorname{Lim}_{t \downarrow a} f(t)$ is nonempty and connected.

Proof. Note that $\Gamma(f)$ is a continuum by [5, p. 17, Theorem 2.5.].

1. Choose any $a \in(x, y]$ and prove that $\operatorname{Lim}_{t \uparrow a} f(t)$ is connected (it is nonempty by Lemma 3.5).

Assume that $\operatorname{Lim}_{t \uparrow a} f(t)$ is not connected. Then there exist nonempty open sets $U, V$ in $\operatorname{Lim}_{t \uparrow a} f(t)$ such that $U \cap V=\emptyset, U \cup V=\operatorname{Lim}_{t \uparrow a} f(t)$. Choose $v \in V$ and $u \in U$. Without loss of generality suppose that $u<v$. If $[u, v] \subseteq \operatorname{Lim}_{t \uparrow a} f(t)$, then $U \cap[u, v]$ and $V \cap[u, v]$ are nonempty, open in $[u, v]$ and $U \cap V \cap[u, v]=\emptyset$ - this means that $[u, v]$ is not connected - a contradiction. Therefore there exists a $s \in(u, v) \backslash(U \cup V)$ such that $(a, s) \in\{a\} \times f(a)$ (since $f(a)$ is connected by assumption).

Note that it follows from Lemma 3.5 that $\operatorname{Lim}_{t \uparrow a} f(t)$ is closed in $f(a)$. Since $(a, u)$ and $(a, v)$ are points in $\operatorname{Lim}_{t \uparrow a} f(t)$, there exist sequences

$$
\left\{\left(x_{i}^{1}, y_{i}^{1}\right)\right\}_{i=1}^{\infty} \in \Gamma(f)
$$

with the limit $(a, u)$ and

$$
\left\{\left(x_{i}^{2}, y_{i}^{2}\right)\right\}_{i=1}^{\infty} \in \Gamma(f)
$$

with the limit $(a, v)$, and $x_{i}^{1}<x_{i}^{2}<x_{i+1}^{1}$ for each positive integer $i$. Since $\lim _{i \rightarrow \infty} y_{i}^{1}=u, \lim _{i \rightarrow \infty} y_{i}^{2}=v$, and $u<s<v$, there exists a positive integer $i_{0}$ such that for each $i \geq i_{0}, y_{i}^{1}<s<y_{i}^{2}$. Recall that the graph of $f$ on each $\left[x_{i}^{1}, x_{i}^{2}\right]$ is a continuum and therefore also connected by [5, p. 17, Theorem 2.5.]. Therefore we can choose a sequence $\left\{\left(x_{i}, s\right)\right\}_{i=i_{0}}^{\infty} \in \Gamma(f)$, where $x_{i} \in\left[x_{i}^{1}, x_{i}^{2}\right]$, such that $\left\{\left(x_{i}, s\right)\right\}_{i=1}^{\infty}$ is convergent with the limit $(a, s)$. This means that $(a, s) \in\{a\} \times \operatorname{Lim}_{t \uparrow a} f(t)$. Recall that $(a, s) \notin\{a\} \times(U \cup V)$. This contradicts the assumption that $U \cup V=\lim _{t \uparrow a} f(t)$.

2. Using a similar arguing we get that $\operatorname{Lim}_{t \downarrow a} f(t)$ is nonempty and connected for each $a \in[x, y)$.

Finally, we introduce countably Markov interval functions.

Definition 3.7. Let $x, y \in \mathbb{R}, x<y$ and let $A$ be a countable subset of $I=[x, y]$, containing the endpoints $x$ and $y$ and such that the derived set $A^{\prime}$ of $A$ is a finite subset of $A$. We say that a u.s.c. function $f$ from $I$ to $2^{I}$ is a countably Markov interval function with respect to $A$, if

1. for each $a \in A$, there exist $u, v \in A$ such that $u \leq v$ and $f(a)=[u, v]$ (degeneracy $u=v$ is possible),

2. the restriction of $f$ on every interval of $I \backslash A$ is an injective singlevalued function,

3. for each $a \in A \backslash A^{\prime}$, the limits $\operatorname{Lim}_{t \downarrow a} f(t), \operatorname{Lim}_{t \uparrow a} f(t)$ are subsets of $A$,

4. if $a \in A^{\prime}$ and $a \neq x$, then $\min \left(\operatorname{Lim}_{t \uparrow a} f(t)\right), \max \left(\operatorname{Lim}_{t \uparrow a} f(t)\right) \in A$; if $a \in A^{\prime}$ and $a \neq y$, then $\min \left(\operatorname{Lim}_{t \downarrow a} f(t)\right), \max \left(\operatorname{Lim}_{t \downarrow a} f(t)\right) \in A$. 
The set $A$ is called the Markov partition for the function $f$.

A function $f$ is a countably Markov interval function if there exists a set $A$, such that $f$ is countably Markov interval function with respect to $A$.

Remark 3.8. Since $A^{\prime}$ is a subset of $A$, obviously $A$ is closed. Minima and maxima that appear in Definition 3.7 do exist since $\operatorname{Lim}_{t \uparrow a} f(t), \operatorname{Lim}_{t \downarrow a} f(t)$ are nonempty and closed by Lemma 3.5 .

Remark 3.9. By Theorem 2.1, each countably Markov interval function has a closed graph, since it is a u.s.c. function.

One can easily see that every (generalized) Markov interval function (as defined in [3] and [1]) is also a countably Markov interval function. The set $A$ is finite, therefore also countable and the set $A^{\prime}$ in this case is empty.

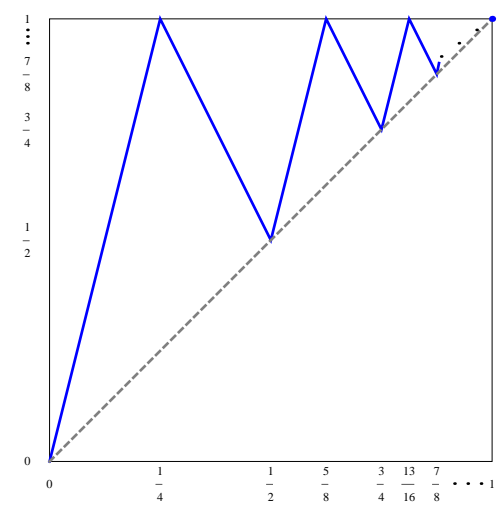

Figure 3. The graph of the function from Example 3.10.

Example 3.10. Let $f:[0,1] \rightarrow 2^{[0,1]}$ be the function from $[6, \mathrm{p} .17]$. The graph $\Gamma(f)$ is the union of the infinite sequence of straight line segments as shown in Figure 3. Obviously, for $A=\{0,1\} \cup\left\{1-\frac{3}{2^{i+1}} \mid i=1,2,3, \ldots\right\} \cup$ $\left\{1-\frac{1}{2^{i}} \mid i=1,2,3, \ldots\right\}, f$ is countably Markov interval function with respect to $A$. The set $A^{\prime}$ is the singleton $\{1\}$.

This is an example of a countably Markov interval function whose inverse limit has already been studied; the example is taken from W. T. Ingram's book [6, p. 17], where it is attributed to R. Bennet [2].

In Lemma 3.5 and Lemma 3.6, we have already shown some properties of $\operatorname{Lim}_{t \uparrow a} f(t)$ and $\operatorname{Lim}_{t \downarrow a} f(t)$ for any u.s.c. function $f$. If $f$ is a countably Markov interval function with respect to $A$ and if $a \in A^{\prime}$, we give a more precise description as follows.

Proposition 3.11. Let $f: I=[x, y] \rightarrow 2^{I}$ be a countably Markov interval function with respect to $A$. For each $a \in A^{\prime}$ the following statement holds true. 
If $a \neq x$ then there exist $u_{1}, v_{1} \in A, u_{1} \leq v_{1}$, such that

$$
\operatorname{Lim}_{t \uparrow a} f(t)=\left[u_{1}, v_{1}\right] \subseteq f(a),
$$

and if $a \neq y$ then there exist $u_{2}, v_{2} \in A, u_{2} \leq v_{2}$, such that

$$
\operatorname{Lim}_{t \downarrow a} f(t)=\left[u_{2}, v_{2}\right] \subseteq f(a) .
$$

Proof. Let $a \in A^{\prime}, a \neq x$.

By Lemma 3.5, $\operatorname{Lim}_{t \uparrow a} f(t)$ is a nonempty closed subset of $f(a)$, and by Lemma 3.6, $\operatorname{Lim}_{t \uparrow a} f(t)$ is connected. Therefore $\operatorname{Lim}_{t \uparrow a} f(t)=\left[u_{1}, v_{1}\right]$ for some $u_{1}, v_{1} \in I$. By Definition 3.7, $u_{1}, v_{1} \in A$, since $u_{1}=\min \left(\operatorname{Lim}_{t \uparrow a} f(t)\right)$ and $v_{1}=\max \left(\operatorname{Lim}_{t \uparrow a} f(t)\right)$.

The claim about $\operatorname{Lim}_{t \downarrow a} f(t)$ can be proved analogously.

In the following definition we introduce when two countably Markov interval functions follow the same pattern. This is done in such a way that for any two generalized Markov interval functions that follow the same pattern (as introduced in [1]) also follow the same pattern (as introduced in Definition 3.12) when being interpreted as countably Markov interval functions.

Definition 3.12. Let $f: I=[x, y] \rightarrow 2^{I}$ be a countably Markov interval function with respect to $A$ and let $g: J=\left[x^{\prime}, y^{\prime}\right] \rightarrow 2^{J}$ be a countably Markov interval function with respect to $B$. We say that $f$ and $g$ follow the same pattern with respect to $A$ and $B$ if there exists an increasing bijective function $\tau: A \rightarrow B$ such that for each $a \in A$ and for all $u, v \in A$,

1. $f(a)=[u, v]$ if and only if $g(\tau(a))=[\tau(u), \tau(v)]$,

2. $\operatorname{Lim}_{t \uparrow a} f(t)=[u, v]$ if and only if $\operatorname{Lim}_{t \uparrow \tau(a)} g(t)=[\tau(u), \tau(v)]$, and

3. $\operatorname{Lim}_{t \downarrow a} f(t)=[u, v]$ if and only if $\operatorname{Lim}_{t \downarrow \tau(a)} g(t)=[\tau(u), \tau(v)]$.

We say that $f$ and $g$ follow the same pattern if there exist Markov partitions $A$ and $B$, such that $f$ is countably Markov interval function with respect to $A, g$ is countably Markov interval function with respect to $B$, and $f$ and $g$ follow the same pattern with respect to $A$ and $B$.

REMARK 3.13. Note that in Definition 3.12 degeneration is possible, i.e. it may happen that $u=v$.

In the following example we show that the function $\tau$ is not necessarily uniquely determined.

EXAMPLE 3.14 . Let $f:[0,1] \rightarrow 2^{[0,1]}$ be a countably Markov interval function with respect to $A=\left\{\frac{1}{2 n} \mid n=1,2,3, \ldots\right\} \cup\left\{1-\frac{1}{2(n+1)} \mid n=\right.$ $1,2,3, \ldots\} \cup\{0,1\}$, defined by its graph, which is the union of the following segments:

1. the straight line segment with endpoints from $(a, 0)$ to $(a, 1)$ for each $a \in A$, 
2. the straight line segment with endpoints from $(a, 0)$ to $\left(a^{\prime}, 1\right)$ for all $a, a^{\prime} \in A$, where $a<a^{\prime}$ and $\left[a, a^{\prime}\right] \cap A=\left\{a, a^{\prime}\right\}$.

Obviously, $A^{\prime}=\{0,1\}$. Let $g:[0,1] \rightarrow 2^{[0,1]}, \Gamma(g)=\Gamma(f)$ and let $B=A$. First we define $\tau_{1}: A \rightarrow B$ to be the identity function. Then we define $\tau_{2}: A \rightarrow B$ by

$$
\tau_{2}(x)=\left\{\begin{array}{ll}
x ; & x=0 \text { or } x=1 \\
\frac{3}{4} ; & x=\frac{1}{2} \\
\frac{1}{2 n} ; & x=\frac{1}{2(n+1)} \\
1-\frac{1}{2(n+2)} ; & x=1-\frac{1}{2(n+1)}
\end{array},\right.
$$

for each positive integer $n$. Obviously, $\tau_{1}$ and $\tau_{2}$ are both increasing bijective functions from $A$ to $B$ satisfying 1., 2. and 3. from Definition 3.12.

\section{MAin RESUlt}

The following theorem is the main result of this paper.

THEOREM 4.1. Let $\left\{f_{n}\right\}_{n=1}^{\infty}$ be a sequence of functions from $I=[x, y]$ to $2^{I}$ which are all countably Markov interval functions with respect to $A$ and let $\left\{g_{n}\right\}_{n=1}^{\infty}$ be a sequence of functions from $J=\left[x^{\prime}, y^{\prime}\right]$ to $2^{J}$ which are all countably Markov interval functions with respect to $B$. If $f_{n}$ and $g_{n}$ follow the same pattern with respect to $A$ and $B$ for each $n$, then $\varliminf_{\longleftarrow}\left\{I, f_{n}\right\}_{n=1}^{\infty}$ is homeomorphic to $\varliminf_{\longleftarrow}\left\{J, g_{n}\right\}_{n=1}^{\infty}$.

Proof. We fix an increasing bijective function $\tau: A \rightarrow B$, having the properties from Definition 3.12. First we construct inductively homeomorphisms $h_{i}: I \rightarrow J$ such that $h_{i}(t)=\tau(t)$ for each $t \in A$ and $h_{i} \circ f_{i}=g_{i} \circ h_{i+1}$ for each positive integer $i$, see the diagram.

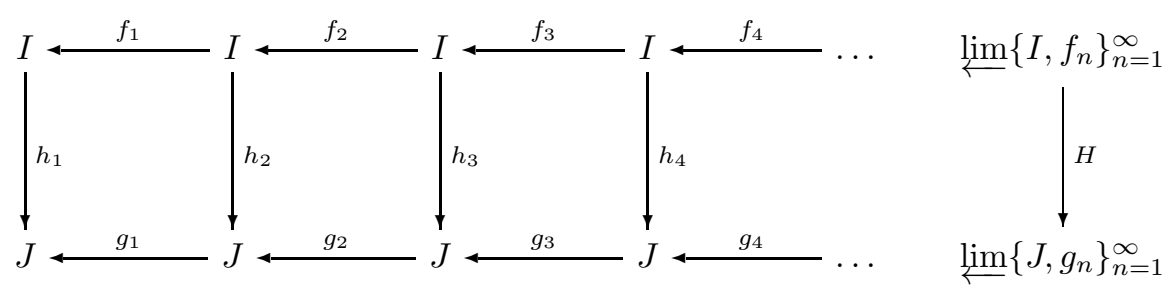

For $i=1$ we fix a homeomorphism $h_{1}: I \rightarrow J$ satisfying the following properties. For any $t \in A, h_{1}(t)=\tau(t)$ and for any interval $\left[a, a^{\prime}\right] \subseteq I$, such that $a<a^{\prime}$ and $\left[a, a^{\prime}\right] \cap A=\left\{a, a^{\prime}\right\},\left.h_{1}\right|_{\left[a, a^{\prime}\right]}$ is a continuous strictly increasing bijective function from $\left[a, a^{\prime}\right]$ to $\left[\tau(a), \tau\left(a^{\prime}\right)\right]$. Obviously, such a homeomorphism exists.

Next we construct $h_{2}: I \rightarrow J$ satisfying

1. $h_{2}(t)=\tau(t)$ for each $t \in A$ and

2. $h_{1} \circ f_{1}=g_{1} \circ h_{2}$ 
as follows:

1. For any $t \in A$, let $h_{2}(t)=\tau(t)$.

2. Let $t \notin A$. Then there exist $a, a^{\prime} \in A$, such that $\left[a, a^{\prime}\right] \cap A=\left\{a, a^{\prime}\right\}$ and $t \in\left(a, a^{\prime}\right)$. Since $f_{1}$ is a countably Markov interval function with respect to $A$, it follows that $\left.f_{1}\right|_{\left(a, a^{\prime}\right)}$ is injective single-valued. Therefore $\operatorname{Lim}_{s \downarrow a} f_{1}(s)$ and $\operatorname{Lim}_{s \uparrow a^{\prime}} f_{1}(s)$ are singletons in $A$, say, $\{\alpha\}=\operatorname{Lim}_{s \downarrow a} f_{1}(s)$ and $\left\{\alpha^{\prime}\right\}=$ $\operatorname{Lim}_{s \uparrow a^{\prime}} f_{1}(s)$. Therefore, if $f_{1}$ is increasing on $\left(a, a^{\prime}\right)$, then $f_{1}(t) \in\left(\alpha, \alpha^{\prime}\right)$, and if $f_{1}$ is decreasing on $\left(a, a^{\prime}\right)$, then $f_{1}(t) \in\left(\alpha^{\prime}, \alpha\right)$. Without loss of generality, we assume that $f_{1}$ is increasing on $\left(a, a^{\prime}\right)$.

Obviously, $h_{1}\left(f_{1}(t)\right) \in\left(\tau(\alpha), \tau\left(\alpha^{\prime}\right)\right)$. Therefore

$$
\emptyset \neq\left. g_{1}^{-1}\right|_{\left(\tau(a), \tau\left(a^{\prime}\right)\right)}\left(h_{1}\left(f_{1}(t)\right)\right) \subseteq\left(\tau(a), \tau\left(a^{\prime}\right)\right) .
$$

Since $\left.g_{1}\right|_{\left(\tau(a), \tau\left(a^{\prime}\right)\right)}:\left(\tau(a), \tau\left(a^{\prime}\right)\right) \rightarrow\left(\tau(\alpha), \tau\left(\alpha^{\prime}\right)\right)$ is bijective, it follows that $\left|\left(\left.g_{1}\right|_{\left(\tau(a), \tau\left(a^{\prime}\right)\right)}\right)^{-1}\left(h_{1}\left(f_{1}(t)\right)\right)\right|=1$, say that

$$
\left(\left.g_{1}\right|_{\left(\tau(a), \tau\left(a^{\prime}\right)\right)}\right)^{-1}\left(h_{1}\left(f_{1}(t)\right)\right)=\left\{t^{\prime}\right\} .
$$

Finally we define

$$
h_{2}(t)=t^{\prime} .
$$

It follows from the construction of $h_{2}$ that $h_{2}(t)=\tau(t)$ for each $t \in A$ and $h_{1} \circ f_{1}=g_{1} \circ h_{2}$. Now we prove that $h_{2}: I \rightarrow J$ is a homeomorphism.

To prove that $h_{2}$ is bijective, it is enough to prove that $\left.h_{2}\right|_{\left(a, a^{\prime}\right)}$ is bijective for each $a, a^{\prime} \in A,\left[a, a^{\prime}\right] \cap A=\left\{a, a^{\prime}\right\}$. The function $\left.h_{2}\right|_{\left(a, a^{\prime}\right)}$ is defined as the composition of three bijective functions, namely, $\left.f_{1}\right|_{\left(a, a^{\prime}\right)},\left.h_{1}\right|_{\left(\alpha, \alpha^{\prime}\right)}$ and $\left(\left.g_{1}\right|_{\left(\tau(a), \tau\left(a^{\prime}\right)\right)}\right)^{-1}$. Therefore $h_{2}$ is bijective.

Since $h_{2}$ is strictly increasing and surjective, it is a homeomorphism.

Let $i>2$ be a positive integer. Assume that we have already defined $h_{1}, h_{2}, \ldots, h_{i}$ such that $h_{k}(t)=\tau(t)$ for each $t \in A$ and $h_{k} \circ f_{k}=g_{k} \circ h_{k+1}$ for each $1 \leq k \leq i-1$. One may construct $h_{i+1}$ similarly as in the above construction of $h_{2}$. More precisely, in the above mentioned construction of $h_{2}$ we just replace 1 by $i$ and 2 by $i+1$. We leave the details to the reader.

Now we define the function $H: \lim \left\{I, f_{n}\right\}_{n=1}^{\infty} \rightarrow \underset{\lim }{\longleftarrow}\left\{J, g_{n}\right\}_{n=1}^{\infty}$. Let $\mathbf{x}=\left(x_{1}, x_{2}, x_{3}, \ldots\right) \in \lim _{\longleftarrow}\left\{I, f_{n}\right\}_{n=1}^{\infty}$. Then $H(\mathbf{x})$ is defined by

$$
H(\mathbf{x})=\left(h_{1}\left(x_{1}\right), h_{2}\left(x_{2}\right), h_{3}\left(x_{3}\right), \ldots\right) .
$$

By [5, Theorem 4.5.], $H$ is a homeomorphism.

\section{REFERENCES}

[1] I. Banič and T. Lunder, Inverse limits with generalized Markov interval functions, Bull. Malays. Math. Sci. Soc. 39 (2016), 839-848.

[2] R. Bennett, On inverse limit sequences, Master's Thesis, The University of Tennessee, 1962.

[3] S. E. Holte, Inverse limits of Markov interval maps, Topology Appl. 123, (2002), 421-427. 
[4] W. T. Ingram, W. S. Mahavier, Inverse limits of upper semi-continuous set valued functions, Houston J. Math. 32 (2006), 119-130.

[5] W. T. Ingram, An introduction to inverse limits with set-valued functions, Springer, New York, 2012.

[6] W. T. Ingram and W. S. Mahavier, Inverse limits, from continua to chaos, Springer, New York, 2012.

[7] J. P. Kelly, Inverse limits with irreducible set-valued functions, Topology Appl. 166 (2014), 15-31.

[8] W. S. Mahavier, Inverse limits with subsets of $[0,1] \times[0,1]$, Topology Appl. 141 (2004), 225-231.

[9] S. Macias, Topics on continua, Chapman \& Hall/CRC, Boca Raton, 2005.

[10] S. B. Nadler, Continuum theory. An introduction, Marcel Dekker, Inc., New York, 1992.

[11] W. Rudin, Principles of mathematical analysis, McGraw Hill Book Co., New YorkAuckland-Düsseldorf, 1976.

M. Črepnjak

Faculty of Natural Sciences and Mathematics

University of Maribor

Koroška 160, SI-2000 Maribor

Slovenia

and

Faculty of Chemistry and Chemical Engineering

University of Maribor

Smetanova 17, SI-2000 Maribor

Slovenia

E-mail: matevz.crepnjak@um.si

T. Lunder

Faculty of Natural Sciences and Mathematics

University of Maribor

Koroška 160, SI-2000 Maribor

Slovenia

E-mail: tjasa.lunder@gmail.com

Received: 12.8 .2016$.

Revised: 16.1.2016. 